\title{
Fission-fragment and neutron data traced back to the macroscopic and microscopic properties of the fissioning systems
}

\author{
K.-H. Schmidt ${ }^{\mathrm{a}}$ and B. Jurado ${ }^{\mathrm{b}}$
}

CENBG, CNRS/IN2P3, Chemin du Solarium, BP. 120, 33175 Gradignan, France

\begin{abstract}
A new model description of fission-fragment yields and prompt neutron emission is developed. The yields of the different fission channels and their properties are attributed to the number of relevant states above the potential-energy landscape on the fission path at the moment of dynamical freeze-out, which is specific to the collective coordinate considered. The model combines well established ideas with novel concepts. The separability principle of macroscopic properties of the compound nucleus and microscopic properties of the fragments strongly reduces the number of model parameters and assures a high predictive power. The recently discovered energy-sorting mechanism in superfluid nuclear dynamics determines the sharing of intrinsic excitation energy at scission and the enhancement of even-odd structure in asymmetric splits.
\end{abstract}

\section{Introduction}

Empirical descriptions of fission-fragment distributions reveal similarities and systematic trends when the nuclear composition or the excitation energy of the fissioning system is varied. One prominent example is the variation of the relative weights and of the properties of the fission channels. Theoretical descriptions, based on the characteristics of the potential-energy landscape on the fission path with the macroscopic-microscopic approach [1-3] and fully microscopic calculations [4] provide some basic understanding of these regularities. However, the ability of theory for quantitative predictions is limited, and the precision of purely theoretical models cannot yet compete with the one obtained with empirical models [5]. But also the applicability of empirical models is rather restricted due to their poor predictive power.

A profound theoretical explanation of these systematic trends is provided by the separability principle [6]. It is based on the results of two-centre shell-model calculations, which revealed that the microscopic properties of the fissioning system beyond the outer saddle are already essentially determined by the nascent fragments [7]. Thus, microscopic properties of the fissioning system can be separately attributed to the fragments, while only the macroscopic properties are specific to the compound nucleus. We exploit the separability principle to empirically deduce the properties of the fissioning system from the body of experimental data and to develop a model, which is intended to combine the high precision of purely empirical descriptions with the predictive power of theoretical models.

\section{Concept}

Our aim is to develop a code for predicting the fission-fragment yields of all the nuclei from polonium to fermium for excitation energies $E^{*}$ ranging from 0 to $15 \mathrm{MeV}$. The code should be easily

\footnotetext{
a e-mail: k.h.schmidt@gsi.de

b e-mail: jurado@cenbg.in2p3.fr
}

This is an Open Access article distributed under the terms of the Creative Commons Attribution-Noncommercial License 3.0, which permits unrestricted use, distribution, and reproduction in any noncommercial medium, provided the original work is properly cited. 
implemented in reactor simulation codes for nuclear-waste transmutation and reactor safety. Recently, the results of a coordinated research project from the IAEA aiming at the development of systematics and nuclear models for the prediction of minor actinides fission yields up to $150 \mathrm{MeV}$ neutron energy have been presented [8]. This report contains various systematics based on fits of several Gaussians to experimental yields. These systematics give very accurate results for regions located very close to the nuclei used to extract the parameters of the fit. Although our code contains a few parameter dependences that have a pure experimental origin, it is mainly based on well founded physical arguments. Therefore, we expect the predictive power of our code to be better than pure systematics in regions where the amount of data is very poor as is the case for minor actinides.

In the same manner as ref. [9], we consider that the features of the fission yields are determined by the sum of the macroscopic potential, given by the liquid drop model (LDM), and shell effects. In the excitation-energy domain of interest for this work the yields are generally interpreted according to the concept of independent fission modes or channels [1]. The different fission modes correspond to specific valleys in the potential energy landscape. One distinguishes between three main modes the 'super long' (SL), 'standard 1' S1 and 'standard 2' S2. The SL mode is characterised by symmetric yields and low total kinetic energy TKE, which can be explained by a strong deformation of both fission fragments at the scission configuration. The standard modes S1 and S2 present asymmetric mass distributions with average masses of the heavy fragments located near 134 and 140, respectively. The asymmetric modes S1 and S2 present higher TKE than the SL mode, the TKE of S1 being 10 to $12 \mathrm{MeV}$ higher than that of S2. This can be understood by a scission configuration with one spherical heavy fragment and a deformed light fragment for S1, and a configuration with two moderately deformed fragments for S2. While the SL channel is associated to the LD macroscopic potential, the two asymmetric standard modes are generally thought to be linked with shells in the heavy fission fragments at $N=82$ and $Z=50$ (spherical shell) for S1 and $N=88$ (deformed shell) for S2 [10]. There are indications for other asymmetric modes but they are less intense than S1 and S2, and their properties are not yet well understood $[11,12]$.

\section{Formulation of the model}

Fission is a dynamical process where the collective degrees of freedom evolve under the influence of the available number of states above the potential-energy surface, friction and inertia. In this work we consider the following collective variables: the deformation of the nascent fragments $\beta_{1}$ and $\beta_{2}$ together with the effective distance between the surfaces of the fragments $d$, which stands for the length of the neck, the mass asymmetry, and the ratio $N / Z$ of each fission fragment. The saddle point is a particular point on the way to fission with limited phase space that justifies the application of the statistical model to estimate the population of the transition states at the fission barrier [13]. Collective variables, which develop slowly compared to the saddle-to-scission time keep a memory of the configuration at saddle. This is expected for the mass-asymmetry degree of freedom, mainly due to the associated large inertia [14]. On the other hand, the $N / Z$ degree of freedom [15] and the deformation [16] have a small inertia and are essentially determined by the available number of states at scission. We consider that the probability for each fission channel is determined by integrals over the level density up to the available energy at the saddle point. This is valid under the assumption of independent fission channels where the potential-energy valleys associated to the different fission modes are well separated when moving from saddle to scission, and the leakage from one mode to the other is excluded. Our model is based on the macroscopic-microscopic approach and the separability principle. The latter principle states that the macroscopic potential depends on the properties of the compound nucleus according to the liquiddrop model, whereas the microscopic potential is fully determined by the number of neutrons and protons in the nascent fragments [6]. The separability principle is the key assumption that allows our model to be predictive over a wide range of fissioning nuclei with a fixed set of parameters. Indeed, thanks to this principle the parameters that describe the shell effects are identical for all fissioning nuclei as they only depend on the mass and charge of the fission fragments.

The determination of the full potential landscape via theoretical calculations is rather complex and, in spite of the progress made, these calculations do not reproduce the observed facts with good precision. Therefore, in our case we extract this information from the available experimental data. 


\subsection{Level density}

Contrary to most of the work done in fission, we use the constant-temperature level density [17]:

$$
\rho\left(E^{*}\right) \propto \frac{1}{T} \exp \left(\frac{E^{*}}{T}\right)
$$

where $T$ is the temperature defined as the average $E^{*}$ per degree of freedom. The available experimental data on level densities reveal a constant-temperature behaviour up to at least $E^{*} \approx 6-7 \mathrm{MeV}$ [18]. It seems that the melting of Cooper pairs as a function of excitation energy causes an almost linear increase of the effective number of nuclear degrees of freedom, which leads to a constant temperature in the superfluid regime [19]. Moreover, it was found recently that the constant-temperature description remains valid up to $20 \mathrm{MeV}$ for medium mass nuclei [20]. In nature this constant temperature behaviour appears in phase transitions (solid-liquid or liquid-gas). Note that the often used Fermi-Gas level density [21] represents the asymptotic behaviour of the level density at high $E^{*}$ where the temperature of the system increases in proportion to $\sqrt{E^{*}}$. Hence, this description is not well adapted to the $E^{*}$ regime considered here.

\subsection{Mass asymmetry}

As said above, in our model the mass yields are determined near the outer fission saddle. To define the nuclear potential in mass asymmetry we assume that the macroscopic potential at saddle follows a parabola whose curvature $C_{L D}$ is given by $C_{L D}=8 K / A_{C N}^{2}$, where $K$ is a parameter that depends on the fissility of the fissioning nucleus $Z_{C N}^{2} / A_{C N}$. This dependence has been experimentally studied and parameterised in refs. [22,23] on the basis of measured mass distributions. According to this parameterisation, the curvature $C_{L D}$ increases linearly with fissility up to $Z_{C N}^{2} / A_{C N}=35$ and then decreases. However, in our study we observed that this decrease fails in describing the transition to symmetric fission of neutron-deficient light actinides. We have found that the latter transition can only be reproduced if the linear increase is maintained for $Z_{C N}^{2} / A_{C N}>35$.

Also in theory, the variation of the stiffness with fissility is a subject of debate: While some models predict a decrease around $Z_{C N}^{2} / A_{C N}=30$ [23], a monotonic increase of the stiffness with fissility was predicted by Nix [24]. Our result seems to support Nix' model. We may suppose that some contribution from quasi-fission or fast fission could be responsible for the increasing widths of the mass distributions of the heavy actinides, which entered into the parameterisation of refs. [22,25]. This problem certainly needs more careful investigation.

We consider only the shell effects that lead to the two asymmetric modes S1 and S2. These shell effects are represented by parabolas whose curvatures and depths are free parameters that are adjusted to the available experimental data. As said above, one generally assumes that the modes S1 and S2 are mainly conditioned by shells in neutron number. Actually, S1 is also the result of the proton shell $Z=50$, although most calculations show that this shell is weaker than the shell at $N=82$. However, very surprisingly, experimental yields show that the mean position of the heavy fragment associated to channel $S 1$ remains very much stable at $\left\langle Z_{S 1}^{h}\right\rangle=52.5$, which implies strong systematic variations in neutron number. Similarly, the mean position of channel S2 stays rather constant at $\left\langle Z_{S 2}^{h}\right\rangle=55$, while the average neutron number varies. This observation has been established for a broad range of fissioning nuclei going from Ac to Cf [29].

When studying this issue in more detail, considering an even larger number of nuclei, we have found a slight dependence of $\left\langle Z_{S 2}^{h}>\right.$ on the mass of the fissioning system. In addition, there are indications that $\left\langle Z_{S 2}^{l}>\right.$ does not exceed the value of 42.4 for the heaviest fissioning nuclei considered. One may assume that the formation of heavier fragments in the light group of the $\mathrm{S} 2$ fission channel becomes energetically unfavoured due to the increased deformation (see next section).

The essentially constant position of the heavy-fragment groups of the S1 and S2 fission channels in $Z$ is a crucial ingredient of our model. The theoretical explanation is not clear. Pashkevich deduced a stabilisation around the 'magic' proton number $Z=54$ at large deformation in his shell-model calculations [26]. However, a deformed proton shell in this region is not found by other authors [10,27]. 


\subsection{Fission-fragment deformation}

The deformation of the fragments is strongly favoured by the mutual Coulomb repulsion of the nascent fragments that induces a considerable elongation of their shapes. The deformation induced in the two nascent fragments can be considered as a superposition of a macroscopic trend given by the LDM, which favours a large prolate deformation around $\beta=0.5$ [10], and a structural influence due to shell effects. As said previously, different fission modes correspond to substantially different deformations at scission. The energy stored in deformation is transformed into $E^{*}$ of the fragments when they snap back to their ground state deformation. The number of neutrons evaporated by each fission fragment is thus very sensitive to the deformation at scission.

Theoretical arguments on the deformation of the fragments at scission can be deduced from shellmodel calculations $[10,27]$. These calculations reveal a very characteristic and systematic feature of shell effects at large prolate deformation: There is a strong correlation between the particle number (neutrons or protons) and an increasing deformation. This feature had already been associated earlier [10] with the variation of the neutron yields as a function of fragment mass. These curves present a saw-tooth-like behaviour with a minimum for the heavy spherical fragment associated to mode S1 and a maximum number of neutrons for the complementary fragment. We considered this feature by assuming linear dependences of $\beta_{1}$ and $\beta_{2}$ with $Z_{1}$ and $Z_{2}$, respectively, which are obtained from experimental data on prompt neutron yields as a function of the fragment mass $v(A)$. The tip distance $d$ is a free parameter whose value is fixed with the help of $v(A)$ data and total kinetic energy (TKE) measurements.

\subsection{Charge polarisation}

Given a scission configuration of two touching emerging fragments $A_{1}$ and $A_{2}$ with deformations $\beta_{1}$ and $\beta_{2}$ and a tip distance $d$, we calculated the macroscopic shape of the potential at scission as a function of the $N / Z$ degree of freedom by taking into account the Coulomb repulsion between the two nascent fragments and the LD potential for each fission fragment. The result is a parabola with a minimum for $N / Z$ that deviates from the value of $N / Z$ of the fissioning nucleus, that is, from the unchanged charge density (UCD) assumption. The deviation with respect to the UCD hypothesis is called charge polarisation.

Polarisation is considerably enhanced due to the shells $N=82$ and $Z=50$ of the S1 mode. Directly measured polarisation data can be used to fix the influence of the $\mathrm{S} 1$ shell on the $N / Z$ degree of freedom. Besides, polarisation depends strongly on $\beta_{1}$ and $\beta_{2}$ and on the tip distance $d$. As mentioned in the previous section, experimental data on $v(A)$ give considerable information on these deformation parameters.

\subsection{Variances}

Once the parabolic shape of the potential for a given degree of freedom is established, the probability distribution for the degree of freedom can be determined by considering the nucleus like an harmonic oscillator in contact with a heat bath of temperature $T$. This leads to a Gaussian distribution with variance:

$$
\sigma^{2}=\frac{\hbar \omega}{2 C} \operatorname{coth}\left(\frac{\hbar \omega}{2 T}\right)
$$

where $\omega$ is the frequency of the harmonic-oscillator potential and $C$ is the stiffness of the potential. At low temperature $T<<\hbar \omega$, expression (2) leads to the width of the distribution corresponding to the quantal zero-point motion: $\sigma^{2}=\frac{\hbar \omega}{2 C}$ At high temperature $T>>\hbar \omega$, eq. (2) gives: $\sigma^{2}=\frac{T}{2 C}$.

However, in the constant-temperature regime, eq. (2) leads to a constant width, and this is in contradiction with the observation that the width of the fission-fragment mass distributions increases with increasing $E^{*}$ [28]. The reason for this discrepancy is that eq. (2) has been obtained by integrating the probability distribution associated to each harmonic oscillator state weighted with the occupation 
probability of each state as given by a Boltzmann distribution. The integration goes up to infinite $E^{*}$, which means that there may be a certain probability to put infinite $E^{*}$ in the degree of freedom. This macro-canonical assumption is valid in classical mechanics for a gas but it is not valid for a nucleus where the $E^{*}$ is limited. In the case of a nucleus, the integral is truncated at the $E^{*}$ of the system and this leads to an increase of the width with $E^{*}$ even in the constant-temperature regime.

In our model we have used the experimental data of [22] to determine the coefficients of the linear relation between $\sigma^{2}$ and $E^{*}$. For the dependence of $\sigma^{2}$ with the mass of the fissioning nucleus for each fission mode we have used the data from C. Boeckstiegel et al. [29]. A recent systematic study of the width and the mean value of the $N / Z$ degree of freedom in fission has been performed in ref. [30].

\subsection{Tunneling}

If the initial excitation energy is close to the fission barrier or even lower, tunneling becomes important. The relative population of the different fission channels is decided by the tunneling through the outer barrier, where the entrance points of the fission valleys in the potential energy of the fissioning system are located. Note that the tunneling through the inner barrier(s) influences the yields of all fission channels in the same way. With the constant-temperature level-density description (1) and the HillWheeler expression for the tunneling, the yields of the different fission channels are obtained by the following integral:

$$
Y_{i}=\int_{0}^{\infty} \frac{1}{T} e^{\frac{\epsilon}{T}} \frac{1}{1+e^{\frac{2 \pi\left(\epsilon+B_{i}-E^{*}\right)}{\hbar \omega_{B}}}} d \epsilon .
$$

It is assumed that the available energy $E^{*}-B_{i}$ above the barrier is shared between intrinsic excitation energy $\epsilon$ and kinetic energy $E^{*}-B_{i}-\epsilon$. Negative kinetic-energy values correspond to tunneling. $B_{i}$ are the heights of the saddle points associated with the different fission channels. The temperature parameter $T$ is defined by the level-density description (see above). We found that the barrier-transmission parameter $\hbar \omega /(2 \pi)$ of $0.3 \mathrm{MeV}$ for all fission channels and all fissioning sytems reproduces the data best. The reason why this value is appreciably larger than those extracted from fission excitation functions is not clear.

\subsection{Excitation energy of the fission fragments}

We assume that near the scission configuration the two nascent fragments have already acquired their individual properties concerning shell effects [7,31,32] and pairing correlations [33] and can be treated as two well defined nuclei set in thermal contact through the neck. We will now consider how the total excitation energy TXE is divided between the two nascent fragments. Following the transition-state approach of Bohr and Wheeler [13], all the available $E^{*}$ above the barrier height is assumed to be thermalised, that means it is equally distributed between all available intrinsic and collective degrees of freedom. The difference in potential energy between saddle and scission [34] may feed some amount of pre-scission kinetic energy in fission direction, excitations of normal collective modes and additional intrinsic excitations. We may distinguish three classes of energy, which add up to the final TXE of the fission fragments at scission:

(i) Collective excitations stored in normal modes

(ii) Intrinsic excitations by single-particle or quasi-particle excitations.

(iii) Deformation energy.

The division of collective excitations among the two fragments is intimately related to the nature of the specific collective mode considered. We have assumed that this energy is equally distributed among the two fission fragments. Direct information on the contribution of collective energy to the $E^{*}$ of the heavy fission fragment can be obtained from the number of neutrons emitted by fragments of mass $A=130$, since this mass corresponds mainly to spherical fission fragments from the S1 channel. The division of intrinsic excitations can be derived when thermal equilibrium is assumed among the intrinsic degrees of freedom in each fragment. As said above, the nuclear level density at low $E^{*}$ is 
very well described by the constant-temperature formula of eq. (1). Egidy et al. have obtained the following dependence of the parameter $T$ of eq. (1) from the nucleus mass number $A$ and from shell effects $S$ from a fit to available data on level densities [18]:

$$
T=\frac{1}{A^{1 / 3}}\left(17.45-0.51 S+0.051 S^{2}\right)
$$

This leads to a very interesting situation for the two nascent fragments at the scission-point configuration: The level density of each fragment is represented by the constant-temperature formula (1) with a specific value of $T$ for each fragment. As a consequence, there is no solution for the division of the intrinsic $E^{*}$ with $T_{1}=T_{2}$. As long as the fragment with the higher temperature is not completely cold, its $E^{*}$ is transferred to the fragment with the lower temperature. That means, a process of energy sorting takes place where all $E^{*}$ accumulates in the fragment with the lower value of the $T$ parameter, while the other fragment looses its entire $E^{*}[35,36]$. According to formula (4), the heavy fragment generally has the lower $T$ and thus attracts all the $E^{*}$. However, due to the influence of shell corrections on $T$, this trend may be reversed if the heavy fragment is stabilised by a strong shell effect. This is expected in the $\mathrm{S} 1$ fission channel, which is characterised by the formation of a heavy fragment close to the doubly magic ${ }^{132} \mathrm{Sn}$. The flow of $E^{*}$ from the hot fragment (the fragment with the higher $T$ ) to the cold fragment (the fragment with the lower $T$ ) can be seen as a way for the entire system made of the two nascent fragments in contact to maximise the number of occupied states. The number of available states of the light nucleus or closed-shell nucleus is small compared to that of the complementary fragment. Therefore, the situation in which the light nucleus or the closed-shell nucleus has part of the $E^{*}$ leads to a smaller number of occupied states than the situation in which the entire $E^{*}$ is transferred to the heavy or the non-closed shell nucleus which offers considerably more available states.

\subsection{Even-odd effect in fission-fragment yields}

A review on the even-odd structures in the fission-fragment yields reveals regular global features as a function of Coulomb parameter and asymmetry [37]. These features are very well reproduced by a dynamical model, which considers the formation of even-even nuclides, generally in the light fragment, as the end process of the energy-sorting mechanism. Details are given in a dedicated article [36]. Even-odd staggering in symmetric splits is described by the model presented in [38].

\subsection{Evaporation}

For the $E^{*}$ domain considered in this work, neutron evaporation from saddle to scission can be excluded. Therefore, we only consider neutron emission from the fission fragments. Neutron evaporation has been modelled taking into account the neutron binding energy of each fission fragment. The neutron kinetic energy is sampled from a Maxwell distribution with the temperature $T$ as given by eq. (4). The latter distribution is truncated in order to satisfy energy conservation. Gamma competition is schematically considered by applying systematically higher effective neutron-separation energies in the case of even-even, even-odd, and odd-even daughter nuclei. This modification flattens out even-odd structures, which are created in the evaporation process.

\section{Parameters}

The most important parameters of the model, which are directly adjusted to the fission data, are listed in table 1 . These 13 parameters, together with the few mentioned already in this text, are most decisive for the results. The description of another 8 parameters, which determine some finer details of the model, would go beyond the scope of this contribution.

The values of most parameters appear reasonable in view of theoretical expectations. Some give new insight into fission and ask for a better theoretical understanding, like the constant positions of the 
Table 1. The values of the most important parameters of the fission model. (The dissipated energy is given as the fraction of the potential-energy release from saddle to scission [34]. See [36] for details of the description of the even-odd effect.)

\begin{tabular}{llll}
\hline fission channels & $Z$ & depth & curvature \\
Standard 1 & 52.5 & $-4.8 \mathrm{MeV}$ & $0.3 \mathrm{MeV} / e^{2}$ \\
Standard 2 & 55 & $-4.0 \mathrm{MeV}$ & $0.1 \mathrm{MeV} / e^{2}$ \\
\hline dissipated energy & fraction & & \\
normal modes & $20 \%$ & & \\
intrinsic & $50 \%$ & & \\
\hline neck distance & $d=1 \mathrm{fm}$ & \\
\hline charge polarization & & \\
enhancement factor & 1.5 & & \\
shift for S1 & 0.5 units & \\
\hline even-odd effect & & \\
threshold & $\frac{T_{1}-T_{2}}{E^{*}}=0.035$ & \\
variance & $0.07\left(T_{1}-T_{2}\right) / \mathrm{MeV}$ & \\
\hline
\end{tabular}

fission valleys in $Z$. A few inconsistencies appear or re-appear, e.g. the insufficient charge polarization obtained by the scission-point model (see below). They suggest further studies.

\section{Comparison with data}

When confronting the model as described above with experimental data, a few systematic deviations showed up. Most of these discrepancies disappeared by applying two modifications, which are described in the following two sub-sections.

\subsection{Relative weight of symmetric fission}

We observed some deviations in the relative weight of the symmetric fission channel, which vary, in most cases gradually, from system to system. We suppose that they originate from weak shells in the fragments. These shells are not strong enough to generate a specific fission channel, but they may modulate the yields as shown in [22]. Since the positions of the symmetric mode in neutron and proton numbers depend on the fissioning system, the shell effect at symmetry might slightly vary. Rather small shell effects in the order of a few $100 \mathrm{keV}$ can explain these deviations.

\subsection{Charge polarisation}

We noticed that the charge polarisation as predicted by the macroscopic scission-point model is not able to reproduce the measured values of the SL and the S2 fission channels. Therefore, we applied an empirical enhancement factor of 1.5 (see table 1). This problem had already been noticed previously [10]. It remains unclear, whether an increase of the neck parameter $d$ as suggested in ref. [10] can solve this problem.

\subsection{Overview}

Figure 1 shows a comprehensive comparison of the modified model with evaluated mass distributions for thermal-neutron-induced fission. The agreement is generally rather good. Only for ${ }^{227,229} \mathrm{Th}$ and ${ }^{237} \mathrm{~Np}$, important discrepancies show up at large yields. Deviations also appear for ${ }^{232} \mathrm{U}$ and ${ }^{254} \mathrm{Es}$ in the tails. However, the involved data points, except for ${ }^{229} \mathrm{Th}$ have very large uncertainties. 


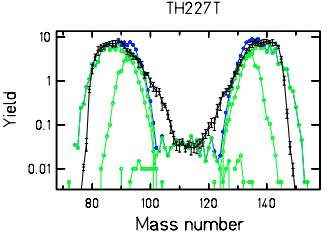

U235T

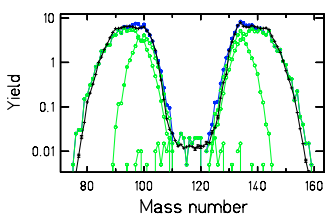

PU242T

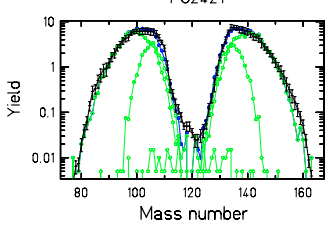

CM245T

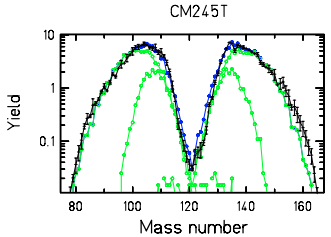

TH229T

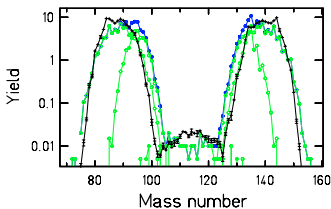

NP237T

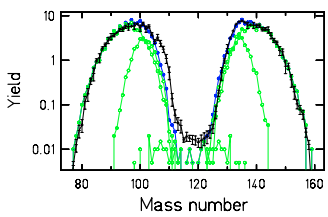

AM241T

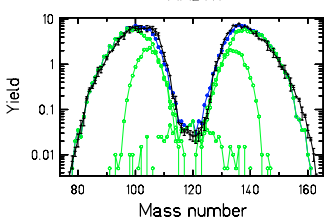

CF251T

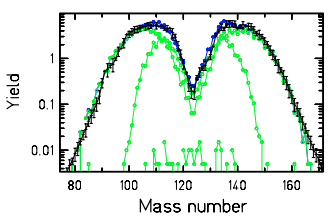

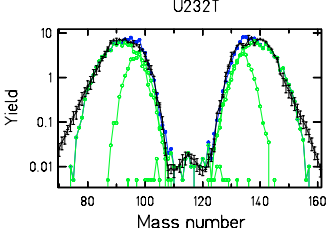

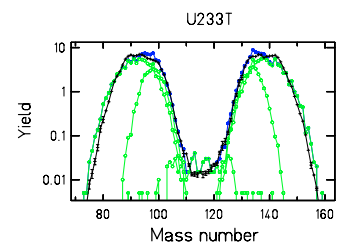

PU239T
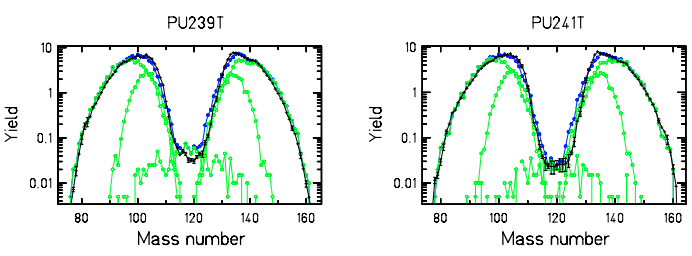

AM242T

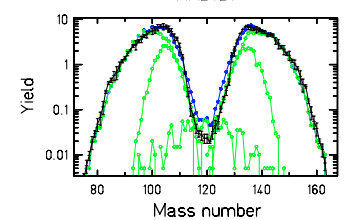

ES254T

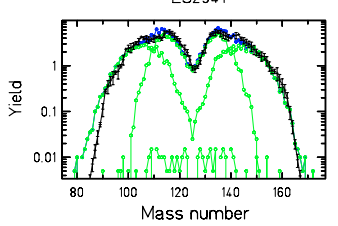

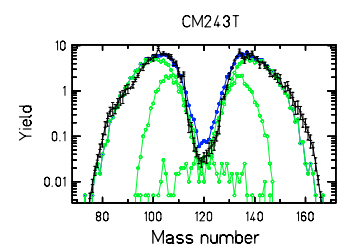

FM255T

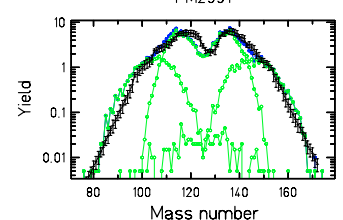

Fig. 1. Comparison of evaluated mass distributions $\left(n_{t h}, f\right)$ [40] (crosses) with results of the GEF code [39] (full symbols). The contributions of the different fission channels are shown in addition (open symbols). The target nuclei are indicated.

\section{Conclusion}

We have elaborated a semi-empirical fission model to predict fission observables for a large variety of nuclei at $E^{*}$ up to $15 \mathrm{MeV}$ [39]. The model is based on the microscopic-macroscopic approach and the separability principle [6]. Thanks to the latter principle, the model is applicable to a wide domain of nuclei with a single set of parameters. The probability distribution of the different degrees of freedom is determined according to the available phase space near the outer saddle or near the scission point. Whether the probability distribution is fixed closer to saddle or closer to scission depends on the inertia of the collective degree of freedom considered.

While all the work done in fission uses the Fermi-gas expression [21] for describing the level density, we use the constant-temperature level density which is best adapted to the $E^{*}$ range considered in this work $[18,20]$. As a consequence, division of intrinsic excitation energy at scission between the fragments is determined by the energy-sorting mechanism, which we have discovered recently [35]. This mechanism also generates a large even-odd effect in the fission-fragment yields at large asymmetry [36].

Once the theoretical basis of the code is established, certain parameters such as the liquid-drop potential stiffness, shell effects or the temperature dependence with nuclear mass and shell corrections are taken from experimental data.

We compared the results of our code with experimental data for a wide range of nuclei ranging from protactinium to fermium isotopes. We considered a broad $E^{*}$ domain going from spontaneous fission to fission induced by $14.7 \mathrm{MeV}$ neutrons. The results of our model for fission fragment mass and charge distributions and for neutron multiplicities agree rather well with the experimental data. 
This work was performed during several short-term visits of K.-H. S. at the CENBG, financed by the EURATOM 6. Framework Programme "European Facilities for Nuclear Data Measurements" (EFNUDAT), contract number FP6-036434. K.-H. S. also thanks the CENBG for warm hospitality.

\section{References}

1. U. Brosa et al., Phys. Rep. 197, (1990) 167

2. P. Moeller et al., Nature 409, (2001) 785

3. M. C. Duijvestijn et al., Phys. Rev. C 64, (2001) 014607

4. H. Goutte et al., Phys. Rev. C 71, (2005) 024316

5. A. C. Wahl, Atom. Data Nucl. Data Tables 39, (1988) 1

6. K.-H. Schmidt et al., Europh. Lett. 83, (2008) 32001

7. U. Mosel, H. W. Schmitt, Nucl. Phys. A 165, (1971) 73

8. Scientific report STI/PUB/1286 (IAEA, Vienna, 2008) ISBN 92-0-115306-6

9. M. G. Itkis et al., Z. Phys. A 320, (1985) 433

10. B. D. Wilkins et al., Phys. Rev. C 14, (1976) 1832

11. S. I. Mulgin et al., Phys. Lett. B 462, (1999) 29

12. F. J. Hambsch et al., Nucl. Data for Science and Technology, Conf. Proc., vol 59, SIF, Bologna, 1997, P. 1239

13. N. Bohr, J. A. Wheeler, Phys. Rev. 56, (1939) 426

14. A. V. Karpov et al., Phys. Rev. C 63, (2001) 054610

15. A. V. Karpov, G. D Adeev, Eur. Phys. J. A 14, (2002) 169

16. P. N. Nadtochy, G. D. Adeev, Phys. Rev. C 72, (2005) 054608

17. A. Gilbert, A. G. W. Cameron, Can. J. Phys. 43, (1965) 1446

18. T. von Egidy et al., Phys. Rev. C 72, (2005) 044311

19. M. Guttormsen et al., Phys. Rev. C 68, (2003) 034311

20. A. V. Voinov et al., Phys. Rev. C 79, (2009) 031301(R)

21. H. A. Bethe, Phys. Rev. 50, (1939) 332

22. S. I. Mulgin et al., Nucl. Phys. A 640, (1998) 375

23. M. G. Itkis et al., Phys. Atom. Nucl. 58, (1995) 2026

24. J. R. Nix, Nucl. Phys. A 130, (1969) 241

25. A.Ya. Rusanov et al., Yad. Fiz. 60, (1997) 773 (Phys. At. Nucl. 60, (1997) 683)

26. V. V. Pashkevich, Nucl. Phys. A 169, (1971) 275

27. I. Ragnarsson, R. K. Sheline, Phys. Scr. 29, (1984) 385

28. IAEA TECDOC-1168 (IAEA, Viena 2000)

29. C. Boeckstiegel et al., Nucl. Phys. A 802, (2008) 12

30. H. Naik et al., J. Phys. G: Nucl. Part. Phys. 30, (2004) 107

31. U. Mosel, H. Schmitt, Phys. Rev. C 4, (1971) 2185

32. J. Maruhn, W. Greiner, Z. Phys. 251, (1972) 211

33. H. J. Krappe, S. Fadeev, Nucl. Phys. A 690, (2002) 431

34. M. Asghar, R. W. Hasse, J. Phys. Colloques 45, (1984) C6-455

35. K.-H. Schmidt, B. Jurado, Phys. Rev. Lett. 104, (2010) 212501

36. B. Jurado, K.-H. Schmidt, Contribution to this workshop

37. F. Rejmund et al., Contribution to this workshop

38. F. Rejmund et al., Nucl. Phys. A 678, (2000) 215

39. The GEF code and additional information are available from www.cenbg.in2p3.fr/GEF

40. T. R. England, B. F. Rider, Report LA-UR-94-3106 (Los Alamos Nat. Lab., 1994) 